\title{
Utilização de dados MODIS para a detecção de queimadas na Amazônia
}

\author{
Rodrigo Alexandre Sbravatti PIROMAL ${ }^{1}$, Roberto Javier RIVERA-LOMBARDI ${ }^{1}$, \\ Yosio Edemir SHIMABUKURO ${ }^{1}$, Antonio Roberto FORMAGGIO ${ }^{1}$, Thelma KRUG ${ }^{2}$ \\ RESUMO \\ O objetivo do presente artigo foi o de avaliar o produto MOD14 quanto à sua performance para detectar áreas queimadas na \\ Amazônia, mais específicamente no norte do Estado do Mato Grosso, considerando o período entre 21/06/2004 e 07/07/2004, \\ comparando os resultados fornecidos pelo MOD14 e interpretações visuais de áreas queimadas obtidas de imagens TM/ \\ Landsat-5. O produto MOD14 detectou 51,58\% do total de áreas queimadas, cujo tamanho médio foi de 83,14 ha; os \\ restantes 48,42\% não detectados tinham tamanho médio de 38,74 ha. O produto MOD14 superestima a quantidade de áreas \\ queimadas ao classificar solos expostos, florestas e outros tipos de coberturas da terra como pixels "hot spots"; além disso, este \\ produto não detecta áreas queimadas de dimensões pequenas $(<100 \mathrm{ha})$, proporcionando erros de classificação em grandes \\ áreas de solos expostos.
}

PALAVRAS-CHAVE: Sensoriamento remoto, Áreas queimadas, Algoritmo de detecção de focos de calor.

\section{Use of MODIS data for detection of burned areas in Amazonia}

\section{ABSTRACT}

The main objective of this paper was to evaluate MOD14 product performance to detect burned areas in Amazonia, more specifically in the north of Mato Grosso State (Brazil), considering the period of 06/21/2004 to 07/07/2004, by comparing the results provided by MOD14 product with visual discrimination of burned areas using TM/Landsat- 5 images. MOD14 product detected $51.58 \%$ of the total burned areas, which have an average size of 83.14 ha; the remaining $48.42 \%$ undetected burned areas have an average size of 38.74 ha. MOD14 product overestimates the burned areas by classifying exposed soils, forests and other types of land cover as hot pixels. In addition MOD14 product does not detect small burned areas $(<100$ ha), generating classification errors in large areas of exposed soils.

KEYWORDS: Remote sensing, Burned areas, Hot pixel detection algorithm.

1 Instituto Nacional de Pesquisas Espaciais - INPE, Caixa Postal 515. CEP 12201-010 - São José dos Campos - SP, Brasil. e-mail: sbravatti@ltid.inpe.br

2 Inter-American Institute for Global Change Research - IAl, Caixa Postal 515 - 12227-010 - São José dos Campos - SP, Brasil. e-mail: thelma@dir.iai.int 


\section{INTRODUÇÃO}

No Brasil, a queima de biomassa vegetal constitui uma prática de manejo utilizada em diferentes culturas, na criação e na manutenção de pastos para criação de gado e na expansão da fronteira agrícola. As queimadas estão amplamente inseridas no processo produtivo da Amazônia e do Cerrado brasileiro e é um fator que impulsiona a expansão agropecuária nestas regiōes. Agricultores e fazendeiros queimam suas terras para converter florestas em áreas de lavouras ou pastagens e para controlar a proliferação de plantas invasoras, de pragas ou de doenças (Coutinho, 1990; Alencar et al., 1997), bem como para estimular a rebrota da pastagem.

As queimadas ocorrem todos os anos durante a estação seca, com maior incidência ao final deste período. Nesta época, quando a vegetação está mais vulnerável ao fogo, fatores tais como o clima seco, o predomínio de gramíneas e outros materiais inflamáveis, expostos a uma fonte de ignição interagem, provocando a ocorrência de queimadas (Coutinho, 1990). Ocasionalmente, fatores de natureza não-antrópica, tais como raios, também são responsáveis pelas queimadas.

A ocorrência de queimadas traz inúmeros impactos ambientais, como o empobrecimento do solo, a perda da biodiversidade de flora e fauna, além de causar prejuízos significativos às propriedades privadas e à sociedade como um todo. Estima-se que as queimadas em regiōes tropicais sejam responsáveis por 32\% da emissão global de $\mathrm{CO}$ para a atmosfera, sendo as queimadas ocorridas na vegetação de Cerrado consideradas como umas das mais importantes fontes de gases traço devido à sua frequiência e extensão (Andreae, 1991).

Os gases traço emitidos durante a queima de biomassa são responsáveis pelo aumento da temperatura e os fluxos do $\mathrm{CO}_{2}$ no solo, resultando em uma perda de carbono para a atmosfera em curto prazo. Estas emissōes, principalmente as de $\mathrm{CO}_{2}$ são responsáveis pelo aumento da absorção da radiação infravermelha pela atmosfera, ocasionando o aquecimento da mesma e, conseqüentemente, do planeta (Kaufman et al., 1994; Klink et al., 1995).

Os registros de ocorrência de queimadas e a análise estatística das mesmas são ferramentas essenciais para se definir estratégias de prevenção e combate a incêndios, além de indispensáveis para assegurar um gerenciamento eficiente e organizado dos mesmos. A distribuição das queimadas ao longo do ano constitui uma informação importante no planejamento da prevenção dos incêndios, pois indica as épocas de maior ocorrência de queimadas, a qual varia significativamente de uma região para outra.

A disponibilidade de informações detalhadas e atualizadas sobre localização e extensão das áreas queimadas é muito importante para avaliar perdas econômicas e efeitos ecológicos, monitorar mudanças no uso e cobertura da terra e elaborar modelos atmosféricos e de impactos climáticos devidos à queima de biomassa vegetal. $\mathrm{O}$ sensoriamento remoto representa uma ferramenta particularmente útil para obter essas informaçôes, principalmente em extensas áreas afetadas pelo fogo e/ou áreas de difícil acesso (Pereira et al., 1997).

Muitas vezes, as estimativas de áreas afetadas por queimadas são geradas a partir do número de focos de calor (indicativo de possíveis fogos ativos) detectados pelas bandas termal $3(3,55-3,93 \mu \mathrm{m})$ e $4(10,5-11,5 \mu \mathrm{m})$ do sensor AVHRR (Advanced Very High Resolution Radiometer), do satélite meteorológico da série NOAA (National Oceanic and Atmospheric Administration). Contudo, o uso deste sensor apresenta certas limitaçôes, já que foi concebido para realizar observaçóes meteorológicas, provocando incertezas nas estimativas de área queimada.

Uma forma de diminuir a incerteza na detecção de superfícies queimadas é utilizando dados de sensores que apresentem características geométricas e radiométricas apropriadas à localização e discriminação de áreas queimadas.

O sensor MODIS a bordo das plataformas Terra e Aqua foi configurado para fornecer dados sobre a dinâmica da biosfera terrestre visando a compreensão dos processos que ocorrem na superfície da terra, nos oceanos e na atmosfera inferior, causadas por atividades antrópicas ou pela própria natureza. Este radiômetro imageador permite a geração de imagens com resolução espacial de $250 \mathrm{~m}$ a $1.000 \mathrm{~m}$ (na regiáo do visível e infravermelho próximo e na região do infravermelho médio e distante, respectivamente) e possui freqüência de revisita entre 1 e 2 dias no Equador. As radiâncias detectadas pelo sensor são quantificadas em classes dinâmicas de 12 bits, o que implica em 4.096 níveis digitais.

Comparando com o AVHRR/NOAA, os dados obtidos a partir do sensor MODIS devem ser mais precisos e adequados ao monitoramento global e regular de superfícies queimadas, uma vez que apresenta melhoria significativa na geometria de aquisição de seus dados e bandas espectrais projetadas para a identificação de fogos ativos com valores de radiância de saturação mais altos.

Outra inovação do sensor MODIS é a disponibilização de produtos elaborados a partir de seus dados brutos. Com isso, a equipe científica dos produtos terrestres MODIS desenvolveu o produto MOD14, que fornece a localização de focos de calor, baseado na emissão dos alvos nos comprimentos de onda de $4 \mu \mathrm{m}$ e $11 \mu \mathrm{m}$. O algoritmo utiliza uma árvore de decisão, baseado nestas faixas espectrais, e gera interpretações com vários níveis de confiança, conforme os ângulos de incidência da fonte e de observação do sensor (Kaufman \& Justice, 1998). 
Neste contexto, o presente trabalho tem como objetivo avaliar o desempenho do algoritmo de detecção de queimadas do produto MOD14 para o período de 21/06/2004 a 07/07/2004, no norte do estado do Mato Grosso.

\section{ÁREA DE ESTUDO}

A área de estudo corresponde àquela compreendida pela cena TM/Landsat de órbita/ponto 226/69, localizada no norte do estado do Mato Grosso, na região centro-oeste brasileira, com coordenadas geográficas no centro da cena de 12० 59' de latitude sul e $55^{\circ} 00^{\prime}$ de longitude oeste, abrangendo uma área aproximada de $34.225 \mathrm{~km}^{2}$ (Figura 1).

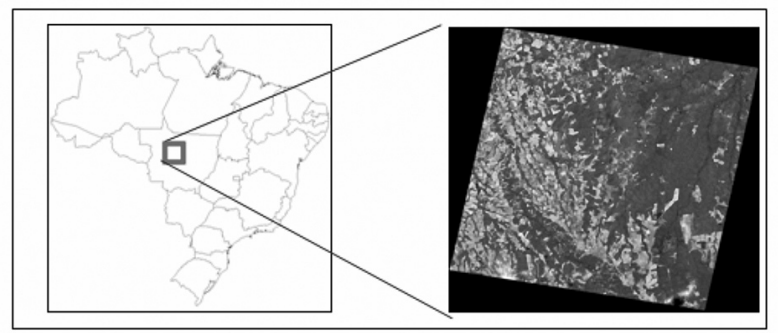

Figura 1 - Localização geográfica da área de estudo e composição colorida (RGB) 4, 5 , 3 da imagem TM/Landsat-5, órbita/ponto 226/69 de 07/07/2004.

As coberturas terrestres dominantes correspondem à floresta umbrófila densa, formações de cerrado e agropecuária extensiva, sendo que as atividades agropecuárias dominam as porçôes centro-sudoeste da área de estudo.

\section{MATERIAL E MÉTODOS}

Para o desenvolvimento do presente trabalho foram utilizados os seguintes dados e aplicativos: (a) duas cenas TM/Landsat-5 de órbita/ponto 226/69, adquiridas em 21 de junho e 07 de julho de 2004, com resolução espacial de 30m; (b) mosaico de imagens TM/Landsat (MrSID) contendo a área de estudo, com resolução espacial de 14,25m; (c) 17 composições diárias (21 de junho a 07 de julho de 2004) do produto MOD14 na projeção Sinusoidal; (d) banco de dados do estado do Mato Grosso na projeção UTM / datum SAD69, contendo: limite estadual, malha municipal, hidrografia principal e secundária, e uso do solo, organizados e processados por Anderson (2004); (e) aplicativo SPRING 4.01 Beta; (f) aplicativo MODIS Reprojection Tool; e (g) aplicativo Geoexpress View.

Foi avaliado o desempenho do algoritmo de detecção de queimadas (MOD14), utilizando como verdade terrestre cicatrizes do fogo discriminadas visualmente a partir de imagens do sensor TM/Landsat. Para tanto, a metodologia adotada no desenvolvimento deste trabalho envolveu as seguintes etapas:

\section{AQUISIÇÃO DE DADOS}

Nesta primeira etapa foram adquiridas as imagens: MODIS do "tile" H12 V10, do produto MOD14 (composição diária), para o período de 21/06/2004 a 07/07/2004; o mosaico TM/ Landsat (MrSid); e as imagens TM/Landsat-5 de órbita/ponto 226/69, nas datas de 21/06/2004 e 07/07/2004.

\section{PROCESSAMENTO DOS DADOS}

$\mathrm{O}$ processamento dos dados envolveu duas fases. $\mathrm{Na}$ primeira, foram realizados os seguintes passos: conversão das imagens TM/Landsat (MrSid) em formato TAR para o formato GeoTiff, utilizando o aplicativo GeoExpress View; conversão das imagens MODIS, produto MOD14 do formato HDF e projeção Sinusoidal, para o formato Geotiff e projeção UTM/WGS84, utilizando o aplicativo MODIS Reprojection Tool; e criação de um banco de dados utilizando o aplicativo SPRING 4.01, onde foram inseridas as referidas imagens. Cabe ressaltar que foi necessário extrair e classificar, mediante a técnica de segmentação de imagens, as áreas correspondentes aos focos de calor contidas no produto MOD14 (Figura 2), uma vez que este produto é gerado como uma matriz de dados espaciais sem atributos de classes. Finalmente, criou-se um mapa temático (mosaico) com os focos de calor referentes ao período de estudo.

$\mathrm{Na}$ segunda fase, as imagens TM/Landsat foram registradas, utilizando como referência o mosaico MrSid. $\mathrm{O}$ registro das duas cenas TM/Landsat foi realizado a partir da seleção de 8 pontos de controle para cada imagem, tendo como base elementos planimétricos estáveis e de fácil identificação, comuns às cenas TM e ao mosaico MrSid. Para o cálculo das coordenadas das cenas utilizou-se um polinômio de transformação de primeiro grau, gerando-se um erro quadrático médio menor que 0.6 pixel $(18 \mathrm{~m})$, em ambas cenas. A transferência dos valores digitais dos pixels para a nova posição corrigida foi realizada adotando o método do vizinho mais próximo, visando preservar os valores radiométricos da cena original.

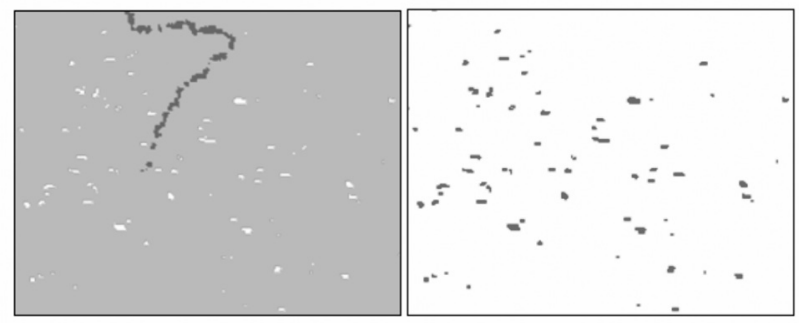

Figura 2 - (a) Focos de calor identificados no produto MOD14; (b) segmentação da imagem em (a). 


\section{INTERPRETAÇÃO DAS CENAS TM/LANDSAT}

Nesta etapa, foram identificadas as áreas queimadas ocorridas durante o período de estudo através da interpretação visual das cenas TM/Landsat diretamente na tela do computador. A utilização da interpretação visual como procedimento para extrair as áreas queimadas foi considerada a mais adequada, uma vez que os procedimentos de classificação digital geralmente confundem padrões espectrais semelhantes, como os apresentados pelas cicatrizes do fogo e outros elementos contidos nas imagens, tais como corpos d'água e sombras de nuvens ou acidentes orográficos. Essas confusōes normalmente geram classificações menos exatas das imagens TM/Landsat do que aquelas resultantes da análise visual (Figura 3).

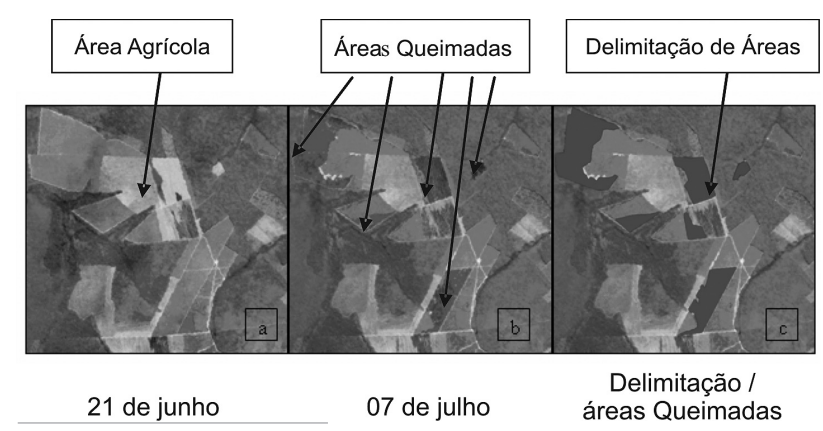

Figura 3 - Imagens TM/Landsat-5 de (a) 21/06/2004, (b) 07/07/2004 e (c) interpretação visual de áreas afetadas por queimadas no período investigado.

\section{ANÁLISE DOS DADOS}

A avaliação do desempenho do algoritmo de detecção de queimadas MOD14, foi realizado através da superposição dos mapas temáticos de áreas queimadas (discriminadas com as imagens TM/Landsat) e dos focos de calor (detectados com o algoritmo MOD14), mediante um procedimento conhecido como álgebra de mapas, utilizando a linguagem de programação LEGAL (Linguagem Espacial para Processamento Algébrico). Este procedimento permitiu verificar se havia ou não correspondência entre pixels rotulados como foco de calor pelo algoritmo MOD14 e pixels classificados como área queimada na imagem TM/Landsat. A partir disto, foi gerado um novo mapa temático contendo pixels de correspondência entre imagens (acertos) e pixels de não-correspondência nas imagens (erros), valendo-se dos seguintes critérios: (a) todo pixel rotulado como foco de calor pelo algoritmo MOD14 e área queimada na imagem TM/ Landsat foi rotulado como Acerto, no novo mapa temático; (b) todo pixel rotulado como foco de calor pelo algoritmo MOD14 e como área não queimada na imagem TM/Landsat foi rotulado como Superestimação no novo mapa temático; e (c) todo pixel rotulado como não foco de calor pelo algoritmo MOD14 e como área queimada na imagem TM/Landsat foi rotulado como Subestimação no novo mapa temático. Assim, o mapa temático foi constituído de três classes distintas: acerto, subestimação e superestimação. Finalmente, foram identificadas visualmente as coberturas terrestres associadas aos erros de superestimação, segundo as classes: solo exposto, queimada anterior ao período investigado, cerrado sensu stricto, agricultura e florestas (Figura 4).

\section{RESULTADOS E DISCUSSÃO}

O mapa temático constituiu-se de 1.102 polígonos, sendo $142(12,88 \%)$ compostos de pixels rotulados como acertos; $286(25,95 \%)$ por pixels rotulados como subestimação e 674 $(61,17 \%)$ por pixels rotulados como superestimação. Assim sendo, polígonos formados por pixels de correspondência entre imagens (acertos) totalizaram 12,88\% do número total de polígonos, enquanto erros (associados à subestimação ou superestimação) totalizaram $87,12 \%$ dos polígonos. A Tabela 1 apresenta a área (em ha) associada aos polígonos de correspondência (acertos) e não-correspondência (erros).

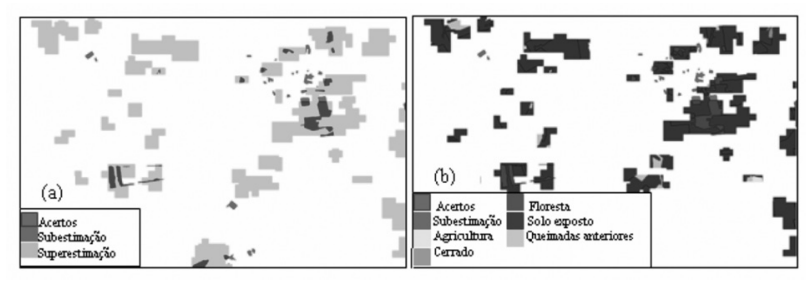

Figura 4 - (a) Mapa contendo polígonos de correspondência (acertos) e não-correspondência (erros); (b) Mapa contendo as coberturas terrestres associadas aos pixels rotulados como superestimação no mapa (a).

Da área total queimada identificada na imagem TM/ Landsat $(22.885,64$ ha), correspondente a Acertos e Subestimação na Tabela 1, somente 11.805,32 ha (51,58\%) foram também identificados pelo algoritmo MOD14. Neste caso, o tamanho médio dos polígonos de concordância foi de 83,14 ha ( $\pm 157,47 \mathrm{ha}$ ). Os restantes $11.080,32$ ha corresponderam a polígonos contendo pixels rotulados como subestimação, sendo o tamanho médio igual a 38,74 ha ( $\pm 70,96$ ha). A rotulação de um pixel como subestimação ocorre quando o mesmo é classificado como área queimada na imagem TM/Landsat e como não-foco de calor no produto MOD14.

Assim, a subestimação ocorre quando as emissōes de radiação eletromagnética dos polígonos de menor área queimada não conseguem sensibilizar os detectores do sensor MODIS.

$\mathrm{Na}$ área de estudo, foram selecionados os menores polígonos de queimadas identificados completamente pelo 
Tabela 1 - Análise das correspondências (acertos e erros).

\begin{tabular}{llllll}
\hline & $\begin{array}{l}\text { Número de } \\
\text { polígonos }\end{array}$ & Área Total & $\%$ & $\begin{array}{l}\text { Área } \\
\text { Média } \\
\text { Polígonos }\end{array}$ & $\begin{array}{l}\text { Erro } \\
\text { Médio }\end{array}$ \\
\hline & - & (ha) & - & (ha) & (ha) \\
Acertos & 142 & $11.805,32$ & 7,88 & 83,14 & 157,47 \\
Subestimação & 286 & $11.080,32$ & 7,39 & 38,74 & 70,96 \\
Superestimação & 674 & $127.020,77$ & 84,73 & 188,46 & 213,64 \\
\hline
\end{tabular}

sensor MODIS, ou seja, áreas em que a área total da queimada foi rotulada como foco de calor, como na Figura 5.

$\mathrm{Na}$ Figura 5, as áreas circundadas em vermelho foram as menores áreas totalmente classificadas como focos de calor pelo sensor MODIS; sendo que, no caso da área à esquerda, a queimada tinha 12,59 ha, o que representa $6,54 \%$ da área total do polígono; já a área à direita, na mesma figura, a queimada tinha 41,12 ha, o que representa $23,77 \%$ da área total do polígono. Desta forma, pode-se afirmar que a menor área completamente identificada pelo sensor MODIS neste trabalho foi de 12,59 ha.

A seguir, pode-se observar na Figura 6 uma distribuição dos polígonos por classes de tamanho, onde é constatado que as áreas superestimadas apresentam uma distribuição mais ampla de tamanhos, enquanto que as áreas identificadas corretamente e principalmente as subestimadas apresentam uma maior concentração em áreas de menor tamanho.

Os dados da Tabela 1 indicam também que um número significativo de polígonos foi constituído por pixels rotulados como superestimação (674), equivalendo a uma área de 127.020,77 ha (84,73\%). O tamanho médio dos polígonos, neste caso, foi 188,46 ha. Uma parte significativa desta superestimação corresponde às classes floresta e solo exposto (43,87 e $47 \%$, respectivamente da área total superestimada), conforme mostrado na Tabela 2. Esse erro decorre do

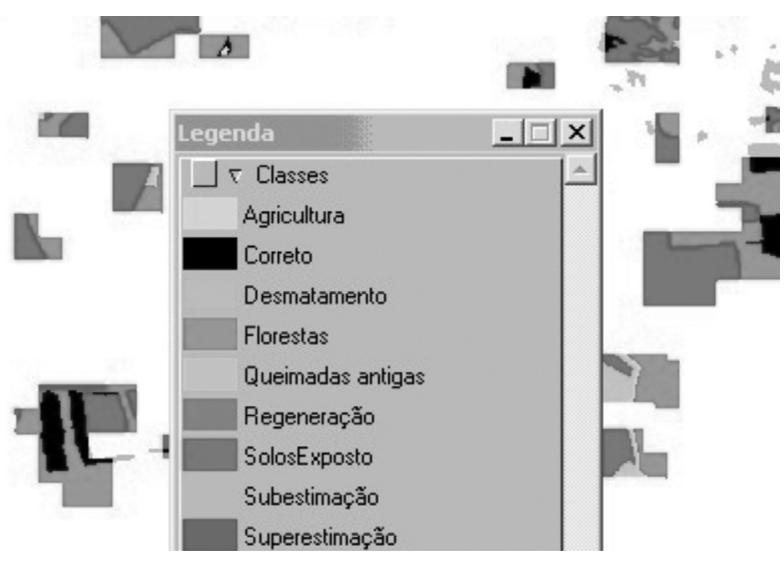

Figura 5 - llustração dos menores polígonos de queimadas identificados pelo sensor MODIS.

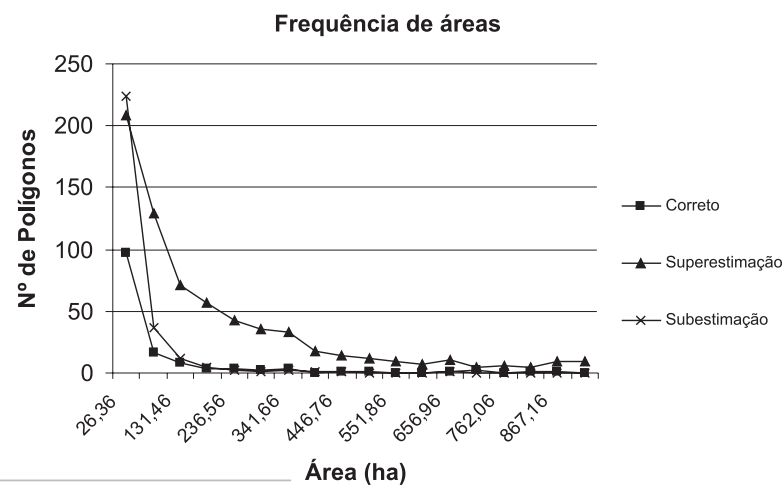

Figura 6 - Distribuição dos polígonos por classes de tamanho, classificação correta, subestimação e superestimação.

fato de as áreas de solo exposto apresentarem a mesma assinatura espectral dos fogos ativos. No caso das florestas, a superestimação está associada à baixa resolução espacial do sensor MODIS e à proximidade destas com as áreas de solo exposto.

Ao se realizar uma análise espacial de vizinhança dos polígonos de concordância e não-concordância, constatou-se que dos 283 polígonos de florestas classificados como focos de calor pelo algoritmo do produto MOD14, apenas 1,77\% (5 polígonos) estavam isolados de áreas de solo exposto, queimadas anteriores ou cerrado. A maior parte dos polígonos de área agrícola apresentava em seu entorno polígonos de solo exposto, queimadas anteriores, e cerrado, além de áreas corretamente classificadas como queimadas.

Segundo Kaufman e Justice (1998), pequenas áreas sendo queimadas, dependendo de sua temperatura, têm a capacidade de saturar o pixel, fazendo com que o mesmo seja classificado como um foco de calor pelo algoritmo do Produto MOD14, mesmo que apenas uma pequena fração da área do pixel esteja sendo queimada.

Este problema também ocorre com o sensor AVHRR/ NOAA. NOAA (1985), Kaufman et al. (1990), Kidwell (1991), Robinson (1991), Setzer (1993) e Chuvieco \&

Tabela 2 - Coberturas terrestres associadas aos polígonos contendo pixels rotulados como Superestimação.

\begin{tabular}{llllll}
\hline $\begin{array}{l}\text { Coberturas } \\
\text { Terrestres }\end{array}$ & $\begin{array}{l}\text { Número } \\
\text { de } \\
\text { polígonos }\end{array}$ & Área Total & $\%$ & $\begin{array}{l}\text { Área } \\
\text { Média } \\
\text { Polígonos }\end{array}$ & $\begin{array}{l}\text { Erro } \\
\text { Médio }\end{array}$ \\
\hline & - & (ha) & - & (ha) & (ha) \\
\hline Agricultura & 30 & $2.882,01$ & 2,27 & 96,07 & 125,91 \\
Florestas & 283 & $55.721,12$ & 43,87 & 196,89 & 212,12 \\
Cerrado & 22 & $3.266,78$ & 2,57 & 148,49 & 154,16 \\
$\begin{array}{l}\text { Queimadas } \\
\text { anteriores }\end{array}$ & 41 & $5.459,08$ & 4,30 & 133,15 & 151,34 \\
Solo Exposto & 298 & $59.691,78$ & 47,00 & 200,31 & 228,91 \\
\hline
\end{tabular}


Martin (1994) apontaram para as seguintes limitações do sensor AVHRR/NOAA: (a) a radiação solar refletida por corpos d'água ou solos expostos, para ângulos específicos de reflexão, podem apresentar a mesma assinatura espectral dos fogos ativos, devido ao baixo limiar de saturação da banda 3 (3,55 a 3,93 $\mu \mathrm{m})$; e (b) embora as bandas $3(3,55$ a 3,93 $\mu \mathrm{m})$ e $4(10,3$ a $11,3 \mu \mathrm{m})$ possam ser utilizadas para identificar a temperatura dos focos de calor, fogos ativos cobrindo apenas parte do pixel $(0,01-0.1 \%)$ são suficientes para saturar a banda 3 deste sensor, superestimando as áreas queimadas.

Comparando-se as situações encontradas na área de estudo com as limitaçôes do sensor AVHRR descritas acima, e considerando que os algoritmos de detecção de queimadas utilizados para o AVHRR e o MODIS são muito semelhantes (utilizando, inclusive, faixas espectrais próximas), pode-se inferir que os problemas acima descritos estão provavelmente ocorrendo de maneira similar com o algoritmo do produto MOD14, mesmo com o aumento da temperatura de saturação nas faixas analisadas. Cabe ressaltar que o AVHRR tem como ponto de saturação $320^{\circ} \mathrm{K}$ para as bandas 3 e 4, e o MODIS possui ponto de saturação de $500^{\circ} \mathrm{K}$ para a banda de $4 \mu \mathrm{m}$ e de $300^{\circ} \mathrm{K}$ para a banda de $11 \mu \mathrm{m}$.

\section{CONCLUSÕES}

Os resultados obtidos neste estudo permitem concluir que o algoritmo de detecção de focos de calor, utilizado pelo produto MOD14, é adequado para identificar queimadas com área próxima ao tamanho nominal dos pixels das imagens MODIS (100 ha). Queimadas com áreas inferiores ao tamanho nominal do pixel não são normalmente detectadas pelo algoritmo.

Observou-se, ainda, que o produto MOD14 classifica erroneamente áreas não queimadas como sendo focos de calor (84,73\% da área). Contudo, pôde-se observar que os erros de superestimação estavam relacionados a alvos que apresentavam comportamento espectral semelhante a solos expostos. A maior parte das áreas $(98,23 \%)$ com comportamento espectral diferente daquele de solos expostos (floresta e agricultura) tinham como vizinhos um ou mais polígonos com comportamento espectral semelhante a solos expostos, e que devido à resolução espacial do sensor e suas características de saturação, foram classificados como focos de calor.

Isso pode estar indicando que a eficiência do MOD14 vai ter maior confiabilidade para detectar focos em áreas menos ocupadas devido a problemas com a vizinhança. Por exemplo, essa ferramenta pode ser útil para localizar invasōes em unidades de conservação e terras indígenas, onde supostamente não haveria problemas com a vizinhança.

Com isso, pode-se dizer que os erros de saturação de pixels e a confusão com áreas de solos expostos apresentados pelo produto MOD14 são muito semelhantes aos gerados pelo sensor AVHRR, apesar do aumento do limiar de saturação para a banda de $4 \mu \mathrm{m}$, e as melhorias nas correçôes geométricas da imagem.

\section{LITERATURA CITADA}

Alencar, A.; Nepstad, D.; Silva, E.; Brown, F.; Lefebvre, P.; Mendosa, E.; Almeida, D.; Carvalho Jr. 1997. O. Uso do fogo na Amazônia: Estudos de caso ao longo do arco de desmatamento. World Bank Report. Brasilia, March.

Anderson, L.O. 2004. Classificação e monitoramento da cobertura vegetal do Estado do Mato Grosso utilizando dados multitemporais do sensor MODIS. Dissertação de Mestrado, Instituto Nacional de Pesquisas Espaciais, São José dos Campos. 247pp.

Andreae, M.O. 1991. Biomass burning: its history, use, and distribution and its impact on environmental quality and global climate. In: Levine, J.S. (Ed). Global biomass burning: atmospheric, climatic, and biospheric implications. Cambridge/ London, MIT Press, Cap. 1, p. 3-121.

Chuvieco, E.; Martín, M. 1994. Global fire mapping and fire danger estimation using AVHRR images. Photogrammetric Engineering \& Remote Sensing, 60(5): 563-570.

Coutinho, L.M. 1990. Fire in the ecology of the Brazilian cerrado. In: Goldammer, J.G., Ed. Fire in the tropical biota. New York, Springer-Velarg, 1990. Cap. 6, p. 82-105.

Crutzen, P.J.; Andreae, M.O. 1990. Biomass burning in the tropics: impacts on atmospheric chemistry and biogeochemical cycles. Science, 250: 1669-1678.

Kaufman, Y.J.; Setzer, A.W.; Justice, C.; Tucker, C.J.; Pereira, M.C.; Fung, I. 1990. Remote sensing of biomass burning in the tropics. In: Goldammer, J.G., Ed. Fire in the tropical biota. New York, Springer-Velarg. Cap. 16, p. 371-399.

Kaufman, Y.J.; Holben, B.N.; Tanré, D.; Ward, D.E. 1994. Remote sensing of biomass burning in the Amazon. Remote Sensing Reviews, 10: 51-59.

Kaufman, Y.J.; Justice, C.O. 1998. MODIS Fire Products. Algorithm Technical Background Document. 77pp.

Kidwell, K.B. 1991. NOAA polar orbiter data (TIROS-N, NOAA-6, NOAA-7, NOAA-8, NOAA-9, NOAA-10, NOAA-11 and NOAA-12): User guide. Washington, DC, NOAA. 1v.

Klink, C.A.; Macedo, R.H.; Mueller, C.C. 1995. De grão em grão o Cerrado perde espaço. WWF-Brasil \& PRO_CER, Brasília.

NASA - National Aeronautics and Space Administration. Earth Observing System Data Gateway. Disponível em http:// edcimswww.cr.usgs.gov/pub/imswelcome/. Acesso em: $12 / 08 / 2004$.

NASA - National Aeronautics and Space Administration. Applied Sciences Directorate. Disponível em http://zulu.ssc.nasa.gov/ mrsid/. Acesso em: 12/08/2004.

NOAA - National Oceanic and Atmospheric Administration. 1985. NESDIS programs - NOAA satellite operations. Wahsington, DC, NOAA. 264pp. 


\section{ACTA}

Pereira, J.M.C.; Chuvieco, E.; Beaudoin, A.; Desbois N. 1997. Remote sensing of burned areas: a review. In: Chuvieco, E.; Ed. A review of remote sensing methods for the study of large wildland fires. Alcalá de Henares. p. 127-183.

Robinson, J.M. 1991. Fire from space: global fire evaluation using infrared remote sensing. International Journal of Remote Sensing, 12: 3-24.

Setzer, A.W. 1993. Operational satellite monitoring of fires in Brazil. International Forest Fire News, 9: 8-11.

Recebido em 12/05/2005

Aceito em 10/01/2008 
